\title{
Pedro José Márquez y la reivindicación de la arquitectura americana
}

\author{
Hilda Julieta Valdés García
}

Artículo

Afiliación: Universidad Nacional Autónoma de México. Ciudad de México, México

E-mail: hildaj@unam.mx

Recibido: 17 de diciembre del 2018

Aceptado: 27 de mayo del 2019

Hilda Julieta Valdés García

Doctora en Letras Clásicas por la Universidad Nacional Autónoma de México. Actualmente es investigadora del Instituto de Investigaciones Bibliográficas de la misma institución.

\section{Resumen}

Tras la expulsión de los jesuitas de España y de sus reinos por Carlos III en 1767, Pedro José Márquez radicó en los Estados pontificios junto con sus hermanos de Orden. En el exilio, los americanos hicieron frente a los agravios raciales de los europeos, que se jactaban de la superioridad de los habitantes del Viejo mundo. En este ambiente hostil hacia los extranjeros, los jesuitas se propusieron defender la patria agraviada en sus escritos.

El presente ensayo dará a conocer la erudición en materia arquitectónica del padre Márquez en el ámbito cultural europeo, así como su audaz respuesta a las ofensas que el prestigioso arquitecto italiano Francesco Milizia emitiera sobre la arquitectura americana a finales del siglo XVIII.

Palabras Clave: Pedro José Márquez; jesuitas novohispanos; Francesco Milizia; arquitectura mexicana; arquitectura peruana

Pedro José Márquez and the assertion of the american architecture

\section{Abstract:}

After the expulsion of Jesuits from Spain and their kingdoms by Carlos III in 1767, Pedro José Márquez settled in the Pontifical States along with his brothers of Order. In exile, the Americans faced the racial grievances of the Europeans, who boasted about the superiority of the inhabitants of the Old World. In this hostile environment towards foreigners, the Jesuits decided to defend the aggrieved homeland in their writings.

This essay will reveal the erudition in architectural matters of father Márquez in the European culture. Moreover, it will expose the audacious response to the offences the prestigious Italian architect Francesco Milizia asserted about American architecture at the end of the 18th century.

Keywords: Pedro José Márquez; novohispanic Jesuits; Francesco Milizia; Mexican architecture; Peruvian architecture 


\section{Introducción}

a llegada de la Compañía de Jesús a Nueva España en 1572 es considerada un parteaguas en la historia de la educación en América. Sin ánimo de denostar la labor evangelizadora y educacional de las otras órdenes religiosas, los jesuitas llegaron al Nuevo Mundo para encomendarles la instrucción de la juventud criolla novohispana, misión que cumplieron hasta 1767, cuando marcharon al exilio por la real orden de expulsión de Carlos III.

Si bien la Compañía se caracterizó por la destreza en la enseñanza del latín, es importante señalar que el plan de estudios o Ratio studiorum comprendía una educación integral y universal. En efecto, se pretendía que el sistema educativo implementado en Europa fuera el mismo en todo el orbe. El éxito de la enseñanza de la lengua latina con los jesuitas se debió, de acuerdo con Pilar Gonzalbo, a la disciplina, a la ejercitación y a la promoción de los cursos (1990, p. 131).

Como se sabe, los jesuitas, nacidos para contrarrestar la Reforma, se esmeraron primero en el dominio de la lengua latina para alcanzar luego la perfección en los estudios retóricos, al grado de reconocer entre sus miembros a varios Cicerones y Quintilianos novohispanos. En las aulas, se promovían debates facticios entre los estudiantes como prácticas de argumentación para enfrentar las invectivas de sus adversarios en la vida cotidiana.

A pesar de los esfuerzos educacionales de los soldados de Cristo, el lenguaje barroco seguía imperando en la Nueva España a mediados del siglo XVIII, por lo que en 1763 se conformó un grupo de sobresalientes ingenios, entre los que se encontraban Rafael Campoy, Diego José Abad, Agustín Pablo de Castro, Francisco Javier Clavijero y Francisco Javier Alegre, iniciadores del movimiento jesuítico renovador que proponía la incorporación del estudio de la lengua griega y de otras lenguas modernas en el currículo. Esto con el fin de acceder a la literatura y a las ciencias experimentales cultivadas en Europa. Para este grupo, era imposible soslayar las ideas de modernidad e ilustración que llegaban al nuevo continente, como lo muestra el Catálogo de la Biblioteca del Colegio de San Pedro y San Pablo de la Compañía de Jesús (Ronan, 1993, pp. 32- 42), cuyos títulos abarcaban todas las áreas del conocimiento.

Acertadamente señalan Ramón Gutiérrez y Cristina Esteras (1993) el papel que jugaron los jesuitas en América: "su ética [...] y su preocupación por los avances científicos en el campo de la geografía, la medicina y las ciencias matemáticas y naturales constituyó para los americanos uno de los centros vitales más poderosos de la modernización barroca" (p. 7).

\section{La voz de los expatriados}

Las inconveniencias de la vida en el destierro no frenaron el espíritu creador e innovador de los jesuitas, quienes se incorporaron al ambiente cultural en los Estados pontificios donde residieron, logrando arrostrar con entereza la denostada imagen que de América se había difundido en el viejo continente a través de las explosivas teorías buffon-depauwianas, en palabras de Antonello Gerbi (1993). En efecto, el ilustrado Georges Louis Leclerc, conde de Buffon (1707-1788), desató la polémica sobre el Nuevo Mundo. En su Histoire naturelle, générale et particulière (1749-1788) había sostenido en los medios académicos que las condiciones ambientales en América conllevaban un proceso degenerativo que impedía a sus habitantes el desarrollo y la civilización. Así, dentro de este esquema, en América las especies eran inferiores; tanto los animales como la vegetación aún no habían alcanzado el grado de madurez. Otro ilustrado, Cornelio de Pauw (1739-1799) llevó al extremo la teoría buffoniana en su obra Recherches philosophiques sur les Américains, ou Mémoires interéssants pour servir à l'histoire de l'espèce humaine (1768), y concluyó que en esta parte del océano, los inhóspitos lugares que había allí explicaban el salvajismo de sus habitantes y su incapacidad intelectual. Por su parte, el historiador escocés William Robertson (17211793), en su The History of America publicada en 1777, secundó también la teoría de 
Buffon sobre la falta de desarrollo en América debido al factor ambiental, por lo que el Nuevo Mundo debía estar subordinado al Antiguo.

Este tipo de injurias había tenido lugar desde el descubrimiento del nuevo orbe; varones ilustres como Alonso de la Vera Cruz, en el siglo XVI, y Juan Zapata y Sandoval, en el $\mathrm{XVII}$, defendieron con la pluma las afrentas. Un siglo más tarde, Juan José de Eguiara y Eguren, con su monumental obra Bibliotheca mexicana, hizo ver a sus detractores que el nivel cultural en la Nueva España podía equipararse o superar a cualquier otra nación (Heredia, 1997). Con la llegada a Italia de miles de jesuitas exiliados, la perenne contienda se recrudeció. Los jesuitas americanos, en su mayoría criollos, promotores de modernidad junto con otros intelectuales, eran conscientes de la realidad que vivían, de su otredad, como la llama Osorio $(1989$, p. 12). Este reconocimiento de saberse diferente de los peninsulares les hizo tolerar la vida en el destierro.

Con todo, a pesar de las circunstancias delicadas inherentes al destierro, los jesuitas desempeñaron un buen papel en la historia, pues debe reconocerse con justicia que la labor realizada en Italia por estos varones fue ejemplar. En patria ajena y con restricción de actividades, los jesuitas humanistas hicieron un esfuerzo por seguir cultivándose a pesar de la estrechez económica; de tal manera prosperaron en sus estudios, que lograron no sólo publicar en Italia "obras de valor excepcional en varias disciplinas" (Heredia, 1988, p. 197), sino también ser reconocidos como varones doctos.

De la producción literaria jesuítica de nuestros compatriotas, se ha destacado siempre el ideal americanista impreso en sus obras. Sin embargo, como hombres de su tiempo se interesaron también por otras áreas del saber: teología, literatura, arquitectura, ciencias naturales, entre otras; en sus obras recogieron y divulgaron tradiciones y conocimientos científicos. De casi toda América surgieron voces que reivindicaron a su patria: los jesuitas chilenos, los quiteños y los mexicanos fueron los más representativos. Saranyana (2005) da cuenta de los autores representativos de cada país y los estudios que de éstos se han hecho; sus aportaciones para la sociedad que los recibió son valiosos, pues el contacto directo con la gente nativa durante su labor evangelizadora y educativa en el Nuevo Mundo les hizo ver la realidad que defendían.

Los autores que sobresalieron fueron el ecuatoriano Juan de Velasco; los chilenos Juan Ignacio Molina y Felipe Gómez de Vidaurre; el guatemalteco Rafael Landívar y los mexicanos Diego José Abad, Francisco Javier Clavijero y Pedro José Márquez. Amén del señero trabajo de Ronan sobre Clavijero (1993); Silvia Navia Méndez-Bonito (2005) ha realizado un estudio comparativo de las obras americanistas de Ignacio Molina y Juan de Velasco con la de Clavijero, quien, como se sabe, difundió en el Viejo Mundo la historia y cultura de los antiguos mexicanos a través de su Storia antica del Messico, considerada la mejor respuesta a las ideas diseminadas por Buffon.

\section{Pedro José Márquez, defensor de la arquitectura americana}

Los biógrafos de Pedro Márquez han lamentado, con justa razón, que fuera mejor conocido lejos de su patria que en ella (Sosa, 1884). Nacido en 1741 en San Francisco del Rincón, Guanajuato, Márquez era estudiante del primer año de teología en el Colegio Máximo de San Pedro y San Pablo de México cuando marchó al destierro junto con sus hermanos de orden. Llegó a Bolonia y luego pasó a Roma. Allí conoció al embajador de España en Italia Don José Nicolás de Azara, quien, a pesar de haber sido partidario de la extinción de la Compañía de Jesús, supo reconocer el intelecto de algunos jesuitas mexicanos como Clavijero y Márquez, a los que benefició con su mecenazgo. Estos dos jesuitas publicaron sus obra s en lengua italiana, quizá a petición de su mecenas y como señal de la modernidad en que se desenvolvían.

Por el mismo Márquez sabemos que su interés por las antigüedades arquitectónicas ocupó sus desvelos y hacia allá encaminó por completo sus estudios, al dedicar su vida plena a decodificar la teoría de la antigüedad del De architectura, al tiempo que reivindicaba al autor romano Marco Vitruvio Polión. La erudición del padre Márquez sobre la única obra sobreviviente de arquitectura clásica debió llamar la atención de Azara, quien, de acuerdo con el testimonio del español y jesuita también Manuel 
Figura 1. Esercitazioni architettoniche sopra gli spettacoli degli antichi, con appendice sul bello in generale. Biblioteca Nacional de México. Foto: Hilda Julieta Valdéz.
Luengo en su Diario, el ministro español favoreció al guanajuatense "con un empeño jamás visto en él" (2010, t. 26, p. 224).

Pedro Márquez convivió en el círculo cultural de Azara con el esteta español Esteban de Arteaga, los pensionados de la Academia de San Fernando en Roma, los arquitectos Silvestre Pérez, Evaristo del Castillo e Isidoro González Velázquez, y con el arquitecto italiano Francesco Milizia, con quien discordaría en ideas estéticas.

Por la crítica de sus contemporáneos y las aprobaciones de los opúsculos que logró publicar, sabemos que los trabajos de Márquez fueron recibidos con aceptación y, gracias a sus publicaciones, obtuvo reconocimiento y llegó a ser socio honorario de varias Academias de Arte. Según Fernández y Sánchez (1988), la Real Academia de Artes de San Fernando lo aceptó como Académico de Honor en 1797, dos años después de que Márquez dedicara a esta Academia su obra Delle case di città degli antichi Romani, secondo la dottrina di Vitruvio. La Academia de San Luis de Zaragoza, a quien dedicó en 1803 su obra Dell'ordine dorico ricerche [...] con Appendice sopra un'antica Tavola di Pozzuolo, lo aceptó ese mismo año. Desconocemos las fechas en que ingresó a las academias italianas de Florencia y de Bolonia, pero en su publicación de 1801 en Madrid se decía ya miembro de éstas. De acuerdo con Antonella Romani (1998), la Academia de San Lucas lo recibió en 1809; un año más tarde lo haría la

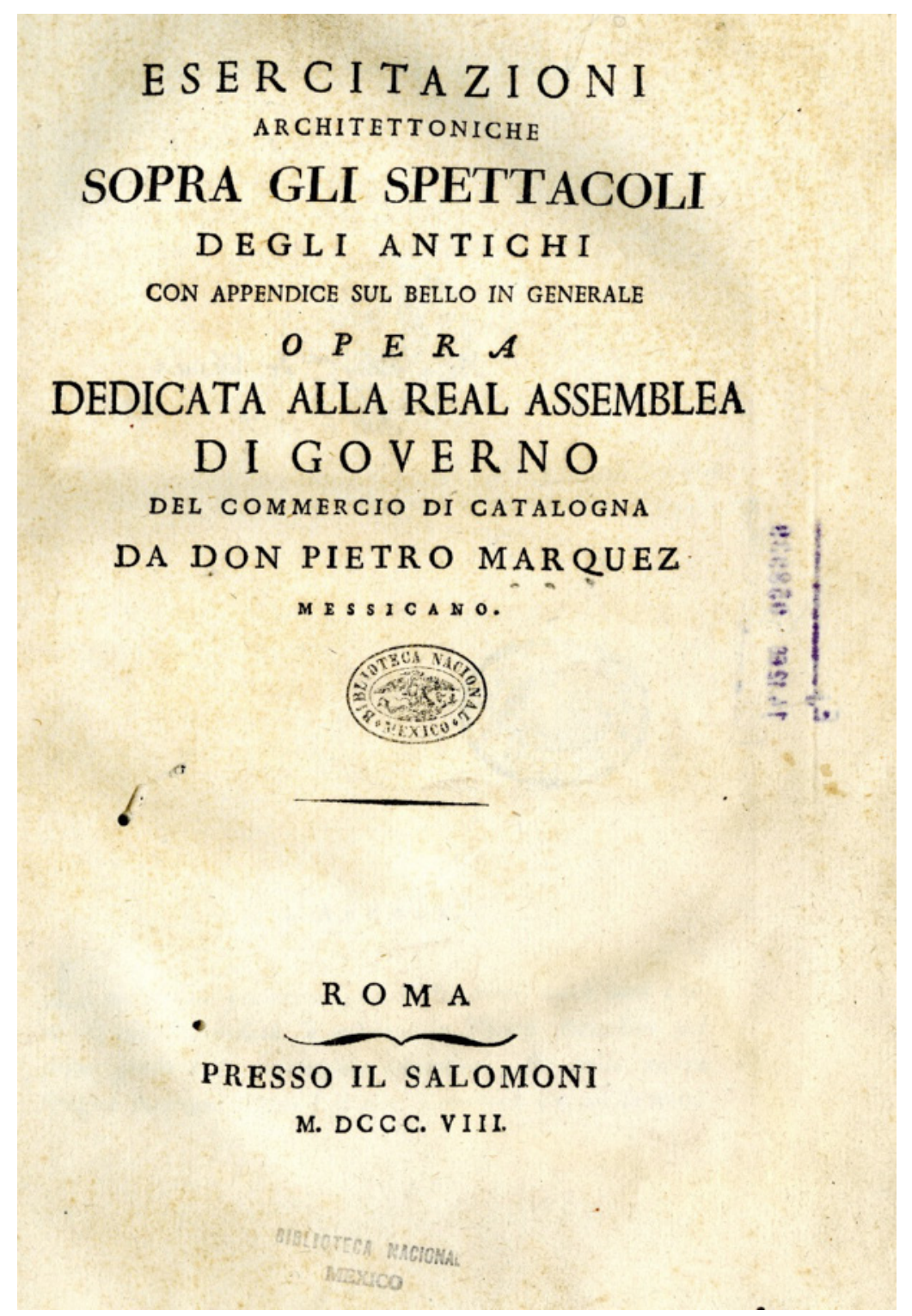

Arqueológica, ambas de Roma. Hay que destacar que, al igual que otros jesuitas, en las portadas de sus obras dejó siempre manifiesto su origen: Pietro Marquez, Messicano (Figura 1).

Los títulos de sus opúsculos y la adscripción honoraria a estas instituciones revelan la erudición alcanzada por Márquez, quien, desde su llegada a Roma en 1780, se sintió atraído por la arquitectura, de modo que pocos años después inició su voluminosa obra Apuntamientos por orden alfabético pertenecientes a la arquitectura, donde se exponen varias doctrinas de $M$. Vitruvio Polión, en que condensó todo el De architectura en forma de diccionario. Esta empresa, iniciada como pasatiempo y en la que invertiría casi tres décadas, llegó a ser una especie de proyecto personal, en el que siguió trabajando. Es de lamentar que nunca llegara a la prensa, tanto por su contenido y orientación didáctica, como por la pérdida de las ilustraciones que lo acompañaban, intituladas Tablas por su autor, y de las que sólo sobrevive un volumen. La portada impresa del manuscrito que 
se conserva -práctica común de la época- evoca la intención de publicarla (Figura 2).

Si bien Gutiérrez y Esteras (1993), en la crítica a la producción bibliográfica de Márquez, reconocieron entre los aportes de Márquez la divulgación de las manifestaciones culturales de los americanos, principalmente "la apertura a las culturas 'centrales' de las manifestaciones arquitectónicas y arqueológicas que existían en América antes de la llegada de los españoles" (pp. 222-223); el recuento biobibliográfico del autor y la selección de fragmentos del lema Arquitectura de los Apuntamientos de Márquez, hacen de este estudio el primer rescate sistemático de su obra inédita. En cuanto a los pasajes sobre la arquitectura americana, destacan los hitos de la arquitectura americana, en especial los correspondientes a la urbanística y la hidráulica.

Inéditos también quedaron algunos textos de Márquez escritos en la madurez y en donde se percibe al autor como experto en la teoría arquitectónica vitruviana (Valdés, 2012). En el discurso Rifflezioni sopra i muri di pietre irregolari, leído muy probablemente en 1811 en la Accademia libera Romana d'Archeologia, actual Academia Nacional de San Lucas, se evidencia la inclinación del erudito mexicano por tocar nuevamente la temática de las construcciones antiguas, encaminadas, sin duda, para insistir en la necesidad de clasificarlas y, justificadamente, ubicar en ellas a las americanas (Valdés, 2014).

En esta disertación, bien elaborada y precisa, Márquez declara en el exordio la imposibilidad de determinar los tipos de muros de piedra irregular. Exalta las construcciones arquitectónicas incas y mexicanas que utilizaron esta estructura. Expone que la construcción de estos muros es común en todo el mundo. Luego, tras manifestar respeto por el interés de los eruditos en dilucidar la datación de los muros de piedra irregular y sus primeros constructores, Márquez argumenta la imposibilidad de concluir la datación, por lo que delimita el tema a desarrollar, y anuncia que sólo tratará de esclarecer el nombre de estos muros.

A continuación, el autor expresa sus divergencias con teóricos contemporáneos como el filólogo y arqueólogo alemán Carl Ludwig Sickler, o sus convergencias con el italiano Andrea Palladio y el español José Ortiz y Sanz. En seguida, debido a que Vitruvio, su autoridad romana, no menciona las piedras irregulares en su tratado, Márquez aduce que el arquitecto romano las contempló en el genus antiquum quod incertum dicitur (género antiguo, llamado incierto), el actual aparejo de mampostería sentado con mortero. Luego, bajo la autoridad de Aristóteles, identifica el género antiguo con la estructura lesbia, que recibe este nombre por el instrumento utilizado para su construcción: la regla lesbia, conocida también como baivel, cercha o escuadra plegable de plomo. De este instrumento, compuesto de dos listones, uno recto y otro curvo, unidos por un extremo a modo de compás, cuyo uso es para arreglar los ángulos mixtilíneos con que se ha de labrar una piedra, Márquez dejó testimonio e ilustración en sus Apuntamientos (Figuras 3 y 4 ).

Figura 2. Apuntamientos o Diccionario vitruviano (1784-1812). Archivo Histórico de la Provincia de México.

Foto: Hilda Julieta Valdéz.

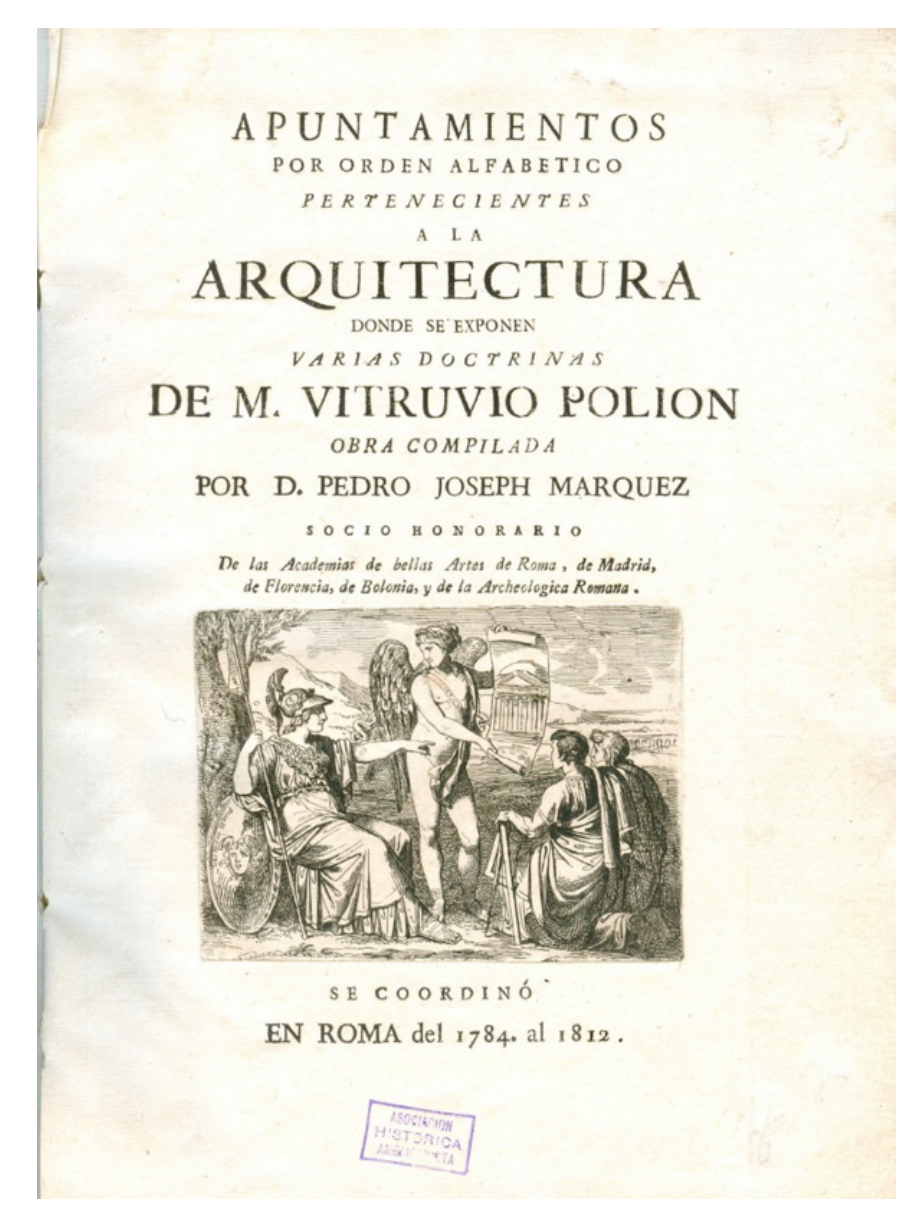


Figura 3. Acotaciones de la Tabla 48. Archivo Histórico de la Provincia de México Baivel: regla cercha o lesbia (f. 2). Foto: Hilda Julieta Valdéz.

Figura 4. Tabla 48 de los Apuntamientos. Archivo Histórico de la Provincia de México Baivel: regla cercha o lesbia (núm. 2) Foto: Hilda Julieta Valdéz.
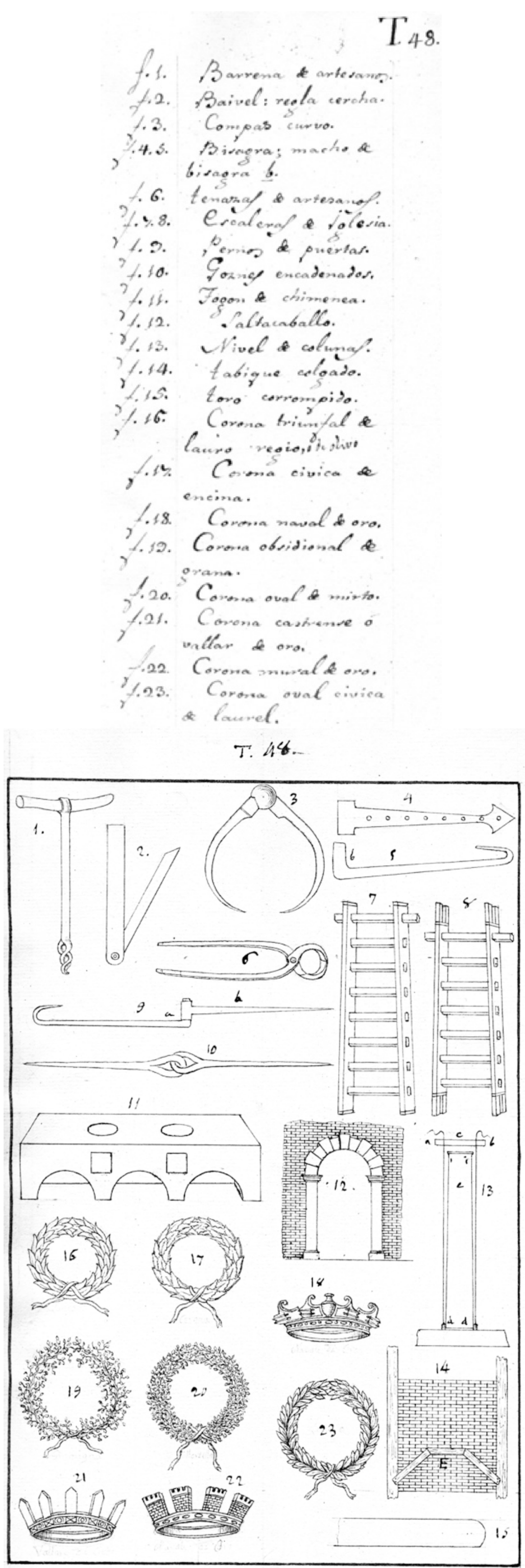

En el último apartado, Márquez distingue la estructura lesbia de la ciclópea (también llamada micénica o pelásgica), pues en la primera, las piedras están unidas con regla y orden; en la segunda, las piedras grandes rústicas están unidas sin pulir.

Las disertaciones sobre las estructuras antiguas y sus nombres eran de suma importancia en plena fiebre anticuaria, pues las exploraciones de las ruinas romanas precisaban el establecimiento de un lenguaje universal, del que, finalmente, se benefició la incipiente 
ciencia arqueológica. Ahora bien, la elección de este tema para una disertación ante el pleno de los miembros de la Academia Arqueológica debe contextualizarse en el interés de un auditorio especializado. En efecto, Márquez, como otros miembros de la Academia, abordó el tema de las estructuras antiguas. Resulta orientador el testimonio de Louis Petit-Radel, miembro de l'Académie des Inscriptions et Belles-lettres y encargado de la Bibliothèque Mazarine, quien dejó una referencia sobre nuestro autor en su obra sobre las construcciones ciclópeas o pelásgicas:

Don Pedro Marquez, architecte mexicain, auteur d'un libre intitulé Des maisons de champagne de Pline le jeune, et don Pedro Velasquez, architecte espagnol, pensionnaire du roi d'Espagne, étaient avec moi dans ma première excursión au mont Circé; le premier me dit qu'il trouvait beaucoup de ressemblance entre les anciens monuments du Pérou et ceux qui étaient sous nos yeux, et je reconnaissais pour cyclopéens. (Petit-Radel, 1841, p. 61)

En varias ocasiones, tanto en la obra impresa como en la manuscrita, el padre Márquez abordó el tema de los materiales y métodos de construcción americanos, con una expresa intención vindicativa.

Los opúsculos de Pedro José Márquez sobre los monumentos antiguos y la doctrina vitruviana son el mejor testimonio de su participación en el ámbito cultural europeo, donde cualquier tema era causa de debate y el acceso a los círculos académicos, muy restringido. Walter Hanisch (citado en Saranyana, 2005) advierte la participación de los jesuitas expulsos en estas acaloradas desavenencias:

A cada rato se levantaban fuertes discusiones sobre todos los temas imaginables. La polémica cundía en Italia por todos los campos del saber hasta llegar a constituir un verdadero género literario, muy propio, por lo demás de la época criticista en que se vivía. Se puede decir que todas las obras tienen en alguna forma este carácter combativo. La política, el derecho, las reformas sociales, la literatura comparada, la historia y la geografía, la filosofía, las ciencias sagradas y las de la naturaleza, todo entraba en el campo de las letras como una réplica a uno o varios escritos. No se limitaba este espíritu a las obras impresas; alcanzaba también a las manuscritas. La vehemencia de los ataques agudizaba por el lenguaje de la época, que consistía en usar la ironía y el sarcasmo como una actitud intelectual frente al adversario. (p. 31)

En este contexto, llama la atención la discrepancia sobre la teoría estética y edilicia entre Pedro José Márquez y Francesco Milizia. El connotado arquitecto italiano no concebía la existencia de monumentos arquitectónicos en el Nuevo mundo; así lo expresaba en su Dizionario delle Belle arti del disegno en su artículo 'Amerciana' (1797, pp. 26-27): "E qual arte di fabbricare in un Emisferio di Sevaggi senza alcun'arte? [...] Gli Americani sono generalmente sì inetti e tanto stupidi da viver fra gli alberi. Stupidi tanto da non saper numerare al di là 20 , e alcuni non pasan il 3".

Si bien Milizia resalta la cultura de México y Perú, los dos imperios más grandes de América, al referirse a las obras arquitectónicas de estas dos capitales, inicialmente pareciera exaltarlas por su realización a pesar de la carencia de herramientas e instrumental: "sapendosi di certo che in tutta America, e al Peru, e al Messico non si conosceva nè ferro, nè calce, nè funi, nè macchine, nè regola, nè compasso, nè buoi, nè cavalli, nè asini" (Milizia, 1797, p. 26); sin embargo, cierra decididamente con la puntilla que con toda seguridad levantó ámpula entre los americanos: "Ma quei del mondo vecchio dovean exagerar tutto nel mondo nuovo. Le grande strade del Perù non erano che stradelle per pedoni, i ponti non eran che salci intralciati, coperti di rami d' alberi. Tutto si faceva a forza di braccia, e forsa inferiormente ai Castori" (Milizia, 1797, p. 26).

Las aseveraciones de Milizia causaron gran indignación, pues este autor debió resultar un incordio para Márquez por la necesaria convivencia en el círculo cultural de Nicolás de Azara. Pensemos que, si como afirma Hanish, todo se debatía, muy probablemente las discusiones entre el italiano y el mexicano se tornaron constantes, al grado de que Márquez describiera a Milizia como un arrogante. Así lo señala en el lema 'Arquitectura' de sus Apuntamientos (Ms. 2456.): "en lo antiguo hubo en América más de lo que parece a ciertos críticos, y que no es exageración todo lo que se lee en las historias de aquellos países, como con su acostumbrado sarcasmo se atreve a decir el Sr. Milizia en tono magistral. La filosofía de que este hombre se precia, no es en muchos puntos 
sino una petulante imprudencia".

Las afrentas padecidas por los americanos en el exilio por la disputa de la supremacía racial de Buffon, pusieron a prueba a los ofendidos. Para Márquez, era claro el pensamiento y actuar del hombre en un ambiente multicultural, así lo expresó: "De tantas naciones que cubren nuestro globo no hay una sola que no se crea mejor que las otras, así como no hay cosa más ordinaria entre los habitantes de la tierra que el burlarse uno de otro cuando lo oye hablar un lenguaje que no es el suyo nativo: efecto de ignorancia que se observa aun en muchos que se tienen por doctos y discretos." (Márquez, 1804, p. III).

Los argumentos de Márquez en defensa de la patria agraviada se encuentran diseminados en sus primeros trabajos sobre antigüedades romanas; donde, a la primera oportunidad, con maestría compara las fábricas arquitectónicas del mundo clásico y el americano, situándolos en igualdad de condiciones, pues el hombre primero emprendió el arte de edificar para cubrir sus necesidades básicas, el embellecimiento de éstas vino después. Así, por ejemplo, a propósito de explicar el pasaje vitruviano 5, 10, 5, sobre el lacónico o baño de vapor romano, trae a cuento el temazcal, su homólogo prehispánico (Valdés, 2008), haciendo hincapié en el uso de la bóveda en América.

Como respuesta a la minusvaloración de Milizia, en más de una ocasión, el padre Márquez dejó registro en sus Apuntamientos de la técnica edilicia inca (Ms. 2456, núm. 52):

Los peruanos, en primer lugar, sin tener noticia de Babilonia, ni haber visto las pirámides de Egipto hicieron obras sorprendentes, solidísimas, y duraderas, todavía existen las ruinas de sus ciudades, de sus palacios, de sus fortalezas, y de sus dilatados caminos: sin el uso del hierro trabajaban, pulían y unían las piedras duras de una especie de granito admirablemente; y sin máquinas las traían de muy lejos, y las subían a diversas alturas; a fuerza de descabezar las barrancas traían las aguas hasta de 200 leguas..

Y al hablar de la Arquitectura mexicana, bajo este vocablo en sus Apuntamientos (Ms. 2456), lo hizo sin perder de vista la tríada vitruviana: utilitas, firmitas, venustas, en la que siempre se apoyaba para explicar la arquitectura prehispánica.

La arquitectura antigua de los mexicanos fue, digámoslo así, la original: ellos conocían los materiales del país y se valían de los mismos oportunamente, hacían obras útiles y sólidas, como eran sus caminos sobre el Lago, Ilamadas hoy Calzadas, que aún se conservan por los españoles, sus acueductos que eran dobles a fin de que cuando se restauraba uno, viniese al agua por el otro, sus templos que eran como una especie de pirámide, a cuya cima, donde estaba la celda del Dios, se subía por muchos escalones; sus palacios que eran grandísimos, habiendo dentro de ellos todo género de comodidades, no sólo de utilidad sino también de lujo, huertos y jardines de flores y yerbas medicinales, serrallos de bestias de todos

Figura 5. Piedra de los doce ángulos. Cuzco, Perú.

Foto: Hilda Julieta Valdéz. géneros, etc. En cuanto a la belleza utilizaban las mejores maderas, ponían en

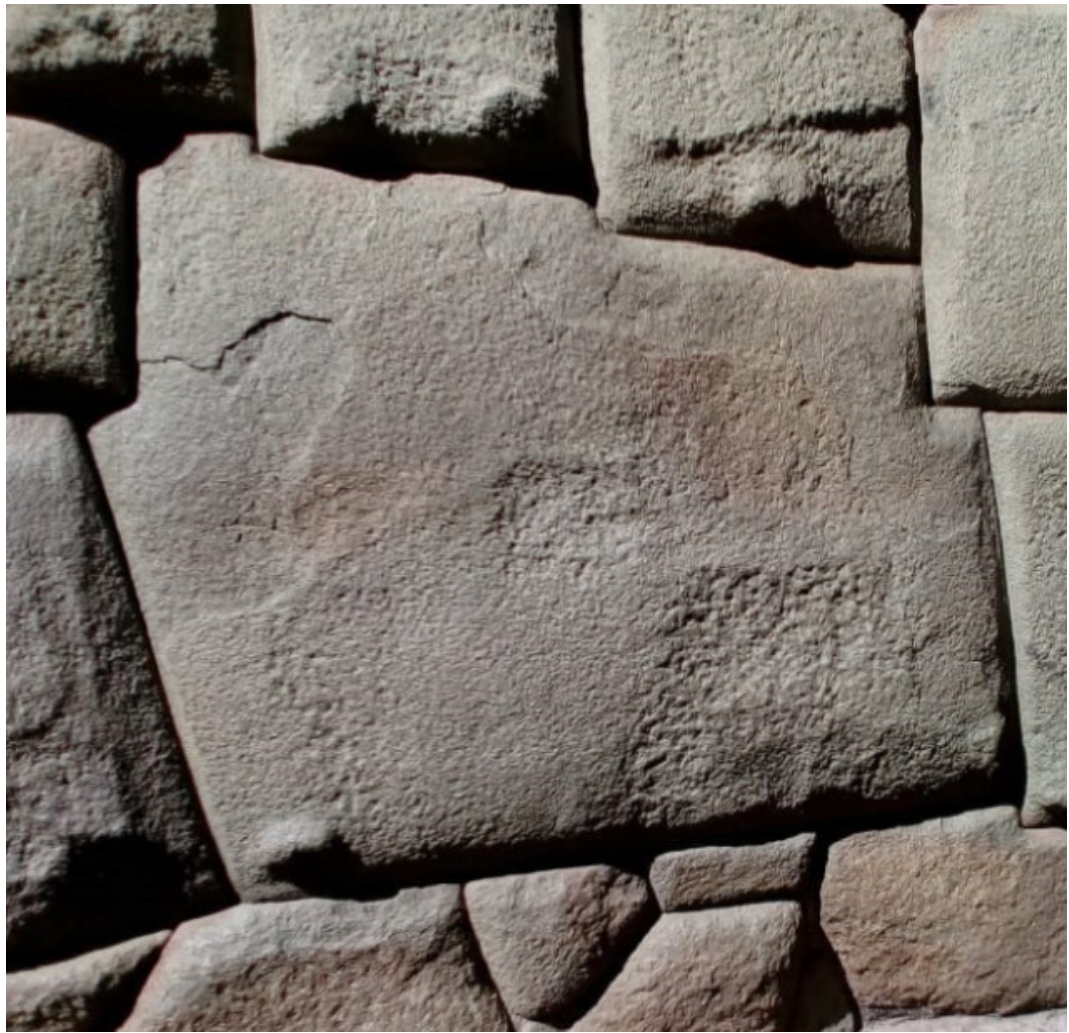


Figura 6. Due antichi monumenti di architettura messicana.

Foto: Hilda Julieta Valdéz. obra algunos mármoles, la plata y el oro, y sus tapicerías eran de algodón o de plumas de pájaros de colores sorprendentes compuestas a lo mosaico. En sus pórticos, que eran muchos, usaban columnas o de madera o de piedra: a más de conservarse algunas de éstas (v. Clavigero).

Tras décadas en el exilio, Pedro José Márquez publicó en 1804 Due antichi monumenti di Architettura messicana. Este texto sobre las pirámides de Tajín y Xochicalco, elaborado a partir de las noticias científicas que de la Nueva España llegaban a Roma. No es de sorprender que con este opúsculo trascendiera la figura de este erudito jesuita en México. La obra fue traducida al español en el 1862 por Lino Ramírez y en 1882 por Francisco del Paso y Troncoso, en el contexto de la construcción de la historia nacional. Fue gracias a Gabriel Méndez Plancarte (1941), quien situó a este jesuita

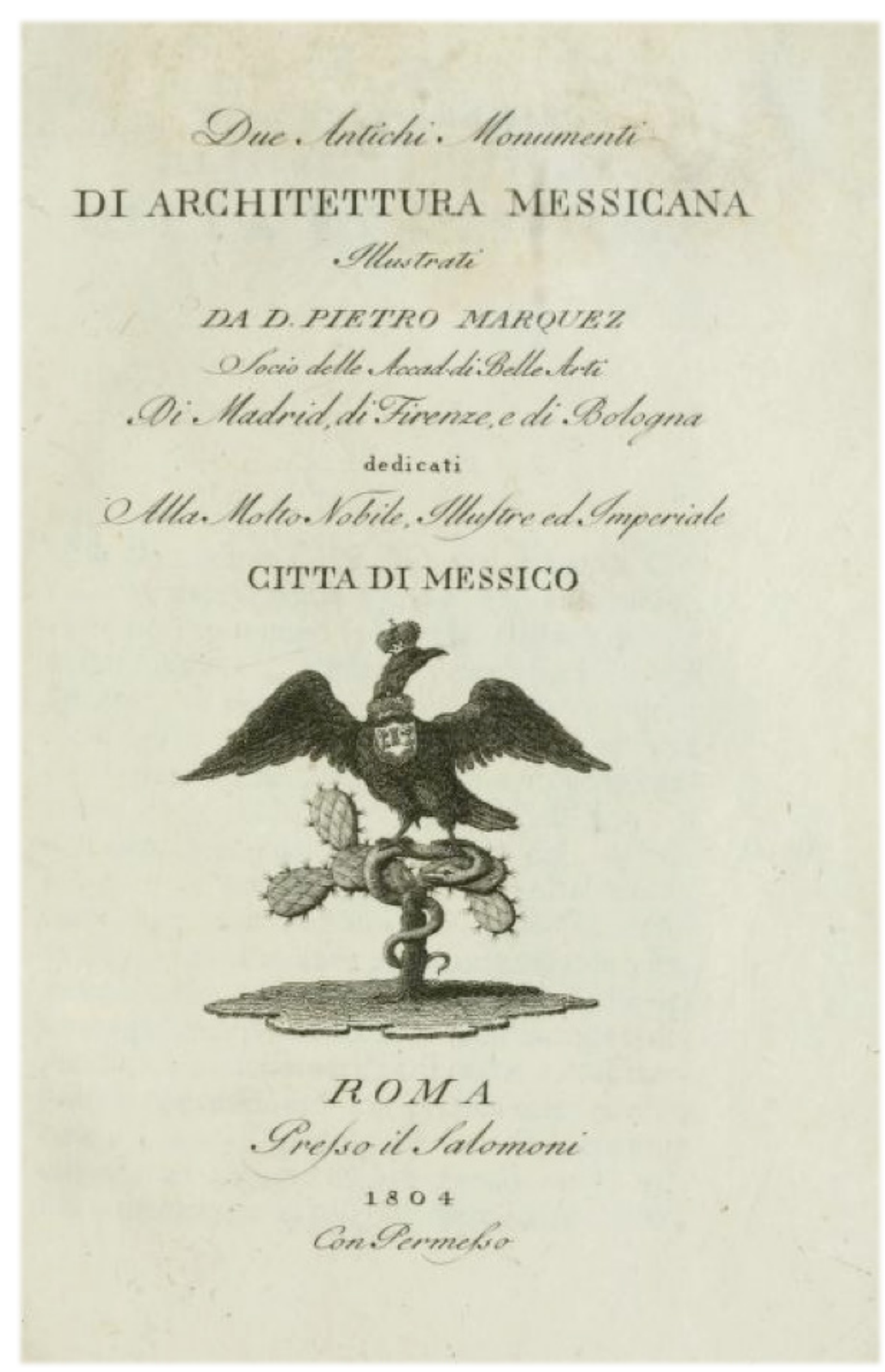

entre los humanistas más destacados del siglo XVIII. Mientras que Justino Fernández (1972) y Juana Gutiérrez Haces (2010) emprendieron sendos estudios de su obra, en particular aquellos donde se pugnaba por el reconocimiento de un arte mesoamericano. Recientemente Carmen Rovira (2014) ha difundido el pensamiento filosófico de Márquez ante la diversidad cultural que le tocó vivir.

\section{Reflexiones finales}

Es muy probable que la participación de Pedro Márquez en los debates sobre las antiguas técnicas edilicias llamara la atención entre los anticuarios dieciochescos; al dar noticia de las fábricas arquitectónicas producidas en América, Márquez se apartaba de la visión eurocéntrica que imperaba en las Academias. La defensa de la arquitectura americana la resumió, al no ser testigo ocular de estas obras, como teórico de la arquitectura, expresando el "no ínfimo grado de civilización y de cultura a que habían ascendido aquellos pueblos mucho antes de que fueran visitados por ningún europeo" (Márquez, 1804, p. IV).

A doscientos años de estas disertaciones, es importante señalar que las investigaciones sobre los métodos y materiales de construcción antiguos continúan vigentes: JeanPierre Adam (2002) es el referente obligado para los tipos de materiales de construcción 
romana; Rabun Taylor (2006) aporta información trascendente sobre las técnicas edilicias utilizadas en los arquitectos del Lacio. Como complemento a las anteriores, Isaac Moreno Gallo (2010) compila técnicas y construcciones en la ingeniería romana, como lo señala el título de su obra, y presenta nuevas investigaciones sobre maquinaria.

Con todo, aunque los avances científicos han logrado dar respuesta sobre la calidad y datación de los materiales pétreos, no ha sucedido lo mismo con la información sobre los métodos y herramientas utilizadas por los antiguos constructores, entre las que sobresale la de los incas en América. Las nuevas tecnologías han permitido a los investigadores exhibir y recrear las construcciones americanas megalíticas de Machu Picchu, Tiahuanaco, Ollantaytambo, Qenqo, Tambomachay y Cuzco, para la construcción hipotética de estos muros resulta ilustrativo el video Exploración inca, de History Channel (2010). Sobre esta arquitectura no sabemos qué admirar más de su enigmática técnica constructiva, si los cortes precisos de las piedras, la destreza del tallado de sus caras hasta hacerlas coincidir perfectamente, el traslado de colosales rocas a elevadas alturas, la nivelación exacta de los acueductos (Figura 4). Tal vez todo en conjunto, principalmente cuando estos monumentos han probado la resistencia de tiempo, la misma que se constata en las obras hidráulicas y en la red viaria romanas.

Finalmente, más allá de destacar el valor que Márquez dio a estas construcciones americanas y la divulgación que a contracorriente hiciera de éstas en el Viejo Mundo, se debe considerar la fortaleza que debió haber cultivado él y sus hermanos de Orden, para sobrellevar en el exilio, sin claudicar, las injurias hacia su persona y su patria, sobre todo si eran cometidas por renombrados personajes de la Europa dieciochesca como Francesco Milizia.

La esencia de esta virtud se encuentra en el pensamiento filosófico de Pedro José Márquez, precisamente en las palabras dedicadas al lector, que introducen el opúsculo Due antichi monumenti di archittetura messicana (1804), y que el jesuita dedicara a la Molto nobile, illustre ed imperiale Città di Messico, por su completa temática americana. Para Márquez el verdadero filósofo es

cosmopolita, es decir, ciudadano del mundo, tiene por compatriotas a todos los hombres y sabe que cualquier lengua, por exótica que parezca, puede en virtud de la cultura ser tan sabia como la griega", y que cualquier pueblo por medio de la educación puede llegar a ser tan culto como el que crea serlo en el mayor grado. Con respecto a la cultura, la verdadera filosofía no reconoce incapacidad en hombre alguno, o porque haya nacido blanco o negro, o porque haya sido educado en los polos o en la zona. tórrida. Dada la conveniente instrucción - enseña la filosofía -, en todo clima el hombre es capaz de todo. (1804, p. III)

En estas palabras se encierra el genuino humanismo cultivado en el exilio por Márquez, quien, como extranjero, supo reconocerse en el otro sin perder su identidad, al tiempo que no se arredró ante los embates de sus adversarios. Esto es el pluriculturalismo para Carmen Rovira (2014), Osorio lo denominó "otredad" (1989, p. 12), con la que los jesuitas sortearon en el infortunio los fenómenos de racismo, xenofobia y migración, que nuestra sociedad globalizada debe combatir.

\section{Referencias bibliográficas}

Adam, J. P. (2002). La construcción romana, métodos y materiales. León: Editorial de los Oficios.

Fernández, J. (1972). Sobre lo bello en general y Dos monumentos de arquitectura mexicana: Tajín y Xochicalco. México: UNAM.

Fernández, M. \& Sánchez, A. (1988). Índice de cargos académicos de la Real Academia de Bellas Artes de San Fernando en el siglo XVIII, Academia, 67, 371-458.

Gerbi, A. (1993). La disputa del Nuevo Mundo. Historia de una polémica 1750-1900. México: Fondo de Cultura Económica.

Gonzalbo, P. (1990). Historia de la educación en la época colonial: la educación de los criollos y la vida urbana. México: El Colegio de México. 
Gutiérrez Haces, J. (2010). El padre Pedro José Márquez, un erudito mexicano en la Italia del siglo XVIII. México: Cuadernos del Seminario de Cultura Mexicana.

Gutiérrez, R. \& C. Esteras (Eds.). (1993). Arquitectura y fortificación de la llustración a la Independencia americana. Madrid: Tuero.

Hanisch, W. (1976). Un ataque dieciochesco a Juan Ignacio Molina. Santiago de Chile: Ediciones "Nihil mihi" (Biblioteca Juan Ignacio Molina, 5).

Heredia, R. (1997). Eguiara y Eguren, las voces concordes. Literatura Mexicana, VIII(2), 511-549.

Heredia, R. (1991). Loa de la Universidad. El "Prólogo" a las Selectae Dissertationes Mexicanae, de Juan José de Eguiara y Eguren, introducción, traducción y notas de. México: UNAM (Biblioteca Hvmanistica Mexicana, 6).

Heredia, R. (1988). José Rafael Campoy, hermano mayor y caudillo. Nova Tellus, 6, 197-220.

History Channel. (2010). Exploración inca. Martin Kweller (Dir.), Felipe Varela (Voz).

Luengo, M. (2010). Diario de 1808: el año de la conspiración. Enrique Giménez e Inmaculada.

Fernández (Eds.). San Vicente del Raspeig: Publicaciones de la Universidad de Alicante.

Moreno, I (Comp.). (2010). Las técnicas y las construcciones en la ingeniería romana. Madrid: Fundación de la Ingeniería Técnica de Obras Públicas.

Márquez, P. (1784-1812). Apuntamientos por orden alfabético pertenecientes a la arquitectura, donde se exponen varias doctrinas de M. Vitruvio Polion (Mss. 2456, 2457, 2458, 2459). Biblioteca Nacional de España.

Márquez, P. (1804). Due antichi monumenti di Architettura messicana. Roma: Presso il Salomoni.

Méndez Plancarte (1941). Humanistas del siglo XVIII. México: UNAM (Biblioteca del estudiante Universitario, 24).

Milizia, F. (1797). Dizionario delle Belle arti del disegno estrattato in gran parte dalla enciclopedia metodica da Francesco Milizia (Tomo I). Bassano, Remondini Tip. ed editore.

Méndez-Bonito, S. (2005). Las historias naturales de Francisco Javier, Juan Ignacio de Molina y Juan de Velasco. En Luis Millones y Domingo Ledezma (Eds.). El saber de los jesuitas, historias naturales y el Nuevo Mundo (pp. 226-250). Madrid: Iberoamericana (Textos i estudios coloniales i de la Independencia, 12).

Osorio, I. (1989). Jano o la literatura neolatina de México. En Osorio (Ed.) Conquistar el eco (pp. 9-49). México: UNAM.

Petit-Radel. L. (1841). Recherches sur les monuments cyclopéens ou pélagiques, et sur leurs rapports avec le plus ancienne civilization de l'Europe. Paris: a l'Imprimerie royal. Recuperado de http://books.google.it/books?id=4U2vOSqiOAMC\&pg= PA1\&hl=it\&source $=g b s \_t o c \_r \& c a d=4 \# v=0$ epage \& $q \& f=f a l s e$

Romani, A. (1998). Pedro José Márquez (1721-1820) e l'immagine del Messico antico nella sua opera sull'archittetura precolombina. Archivum Historicum Societatis lesu, 67(133), 131-159.

Ronan, Ch. (1993). Francisco Javier Clavijero, S. J. (1731-1787), figura de la ilustración mexicana; su vida y obras. Guadalajara: Universidad de Guadalajara-Instituto Tecnológico de Estudios Superiores de Occidente.

Rovira, C. (2014). Humanismo y pluriculturalismo en Pedro José Márquez. En O. Flores (Coord.), El clasicismo en la época de Pedro José Márquez (1741-1820). Arqueología, filología, historia y teoría arquitectónica (pp. 459-464). Madrid: RABASF-IIE.UNAM.

Saranyana, J. (2005). Génesis de los ideales americanistas. En Saranyana, J. (Dir.); Alejos, C. (Ed.).Teología en América Latina (Vol. II/1): Escolástica barroca, Ilustración y preparación de la Independencia (1665-1810) (pp. 706-741). Madrid: 
Iberoamericana-Vervuert.

Taylor, R. (2006). Los constructores romanos. Un estudio sobre el proceso arquitectónico. Madrid:Akal.

Sosa, F. (1884). Biografías de mexicanos distinguidos. México: Oficina Tipográfica de la Secretaría de Fomento.

Valdés, H. (2008). De los baños romanos al temazcalli prehispánico: la interpretación de Vitruvio V, 10 por Pedro José Márquez (1741-1820). Nova Tellus, 26(2), 251270.

Valdés, H. (2014) Sopra le antiche strutture, un discurso inédito de Pedro José Márquez. En 0.

Flores (Coord.), El clasicismo en la época de Pedro José Márquez (1741-1820). Arqueología, filología, historia y teoría arquitectónica (pp. 527-534). Madrid: RABASF-IIE.UNAM.

Valdés, H. (2012). Vitruvio en la obra arqueológica inédita del jesuita mexicano Pedro José Márquez (1741-1820) (Tesis doctoral). Universidad Nacional Autónoma de México. 